\title{
Field Induced Singlet Exciton Dissociation and Exciton-Exciton Annihilation in MEH-PPV Films Studied by Photocurrent Spectra
}

\author{
J. Petrović ${ }^{a, *}$, P. Matavulj ${ }^{a}$, L. Pinto ${ }^{b}$ And S. Živanović Š́telmić ${ }^{b}$ \\ ${ }^{a}$ School of Electrical Engineering, University of Belgrade, Serbia \\ ${ }^{b}$ Institute for Micromanufacturing, Louisiana Tech University, Ruston, LA 71272, USA
}

\begin{abstract}
In this paper we investigate different processes which lead to charge carrier generation in ITO/PEDOT:PSS/MEH-PPV/Al photodetector. Primary photogenerated singlet excitons contribute the photocurrent directly through dissociation on the electrodes. Singlet exciton distribution in the active area of the photodetector is calculated on the base of continuity equation including exciton generation, diffusion, and recombination. Depending on the applied electric field and incident photon flux secondary charge carrier generation processes appear in the active MEH-PPV film. Two bulk charge generation mechanisms are considered. Nonlinear recombination of excitons through exciton-exciton annihilation leads to electron and hole polaron generation. Exciton states are also depopulated by exciton dissociation in the presence of high electric field. We assume hole polarons to be majority charge carriers. Polaron contribution to photocurrent is calculated by solving the continuity and drift-diffusion equations for these carriers. Photocurrent spectra are measured for different bias voltages and different incident photon flux intensities. A good agreement between experimental and simulated data confirms our theoretical approach.
\end{abstract}

PACS numbers: 72.20.Jv, 72.40.+w, 72.80.Le

\section{Introduction}

In the past few decades conductive polymers have shown a great potential for modern electronic and optoelectronic applications. Almost all optoelectronic devices such as light-emitting diodes, solid-state lasers, photodetectors, photovoltaics, and integrated optoelectronic devices have been successfully realized in polymer technology [1]. The performance of these devices is very close or even comparable to their inorganic counterparts. Due to its solubility and absorption in the visible range one of the commonly used conductive polymers is $\operatorname{poly}(2$ metoxy-5-(2'-ethyl-hexyloxy)-1,4-phenylene vinylene) or MEH-PPV. The photophysics of this conductive polymer has been widely studied $[2-6]$. However, because of the great variety of the experimental conditions and processing parameters, unified picture has not been accomplished yet. For many applications the formation of the so-called interchain species (aggregates, excimers, polaron pairs) appears to be important $[3,4,6]$. These species are formed after photoexcitation predominantly through secondary processes (field induced exciton dissociation, exciton-exciton annihilation). Interchain excitations are not preferred in polymer LED devices because they quench the luminescence but they are desir-

* corresponding author; e-mail: jovana@etf.rs able in photodetectors and solar cells as the precursors for free carrier generation. The possibility of controlling the interchain species generation under different conditions could be the key step for further improvement in polymer optoelectronics.

The photocurrent spectra of our ITO/PEDOT:PSS/ MEH-PPV/Al photodiode (ITO and PEDOT:PSS stand for indium tin oxide and poly(3,4-ethylenedioxythiophene): poly(styrenesulfonate), respectively) measured under different bias voltages and for different incident photon fluxes give a good insight in the polymer film photophysics. By theoretical modeling of the photocurrent spectra we were able to quantify the polaron generation process depending on the applied electric field. Simulated photocurrent spectra show excellent fit to our experimental results.

\section{Experimental details}

ITO/PEDOT:PSS/MEH-PPV/Al device was fabricated at Louisiana Tech University on the ITO coated glass substrate. A $150 \mathrm{~nm}$ thick ITO layer coated with water soluble PEDOT:PSS thin film was used for the anode. An active MEH-PPV film was spin-coated from the $10 \mathrm{mg} / \mathrm{ml}$ chlorobenzene solution. Film thickness was $105 \mathrm{~nm}$. A top aluminum electrode $(300 \mathrm{~nm})$ was thermally evaporated on the MEH-PPV film. 
Polymer photodiode was illuminated by the monochromator light. The incident photon flux was changed by applying different optical filters with light transmittance $50 \%, 20 \%, 10 \%$ and $1 \%$. Photocurrent was recorded for each of them in the light wavelength range $370-800 \mathrm{~nm}$ with a $10 \mathrm{~nm}$ step. Reverse bias voltage was tuned from $0 \mathrm{~V}$ to $10 \mathrm{~V}$ with the step size of $0.1 \mathrm{~V}$.

\section{Theoretical basis and the model}

The study of conductive polymers is a rather difficult and challenging task in general. Although from the discovery of these materials in 1976 intensive experimental and theoretical research is in progress, many of the results in the literature are still controversial. Focusing our attention on MEH-PPV it has been shown that photophysics of this polymer is very dependent on film morphology [4]. All the processes that follow photoexcitation are strongly influenced by many physical quantities such as incident photon flux intensity, light wavelength, electric field intensity, and others. After the photoexcitation primary photoexcited states are formed. These states are predominantly singlet intrachain excitons [6], but depending on the degree of the interchain interaction (film morphology) some interchain species can also be primarily generated. Interchain species with different degree of charge separation such as aggregates, excimers, or polaron pairs are possible to apear [3, 4]. Most of the interchain states are populated during secondary processes via energy transfer from ordinary singlet excitons. This can occur through annihilation process of two singlet excitons (exciton-exciton annihilation) if the density of excitons reaches a sufficient level, usually greater than $10^{17} \mathrm{~cm}^{-3}[5]$. The density of singlet excitons is directly related to incident photon flux intensity. If the electric field is applied to photoexcited polymer, the interchain states can be formed from dissociation of singlet excitons [7].

The performance of polymer photodetectors is strongly affected by the number of photogenerated interchain species because they are transients for free charge generation which lead to photoconductivity. After illuminating ITO/PEDOT:PSS/MEH-PPV/Al photodiode singlet excitons are formed by photoexcitation (only $1 \%$ primary generated states could be of interchain type [6]). Singlet excitons directly contribute the photocurrent by dissociation on the electrodes (we neglect dissociation on defects and impurities) to which they travel by diffusion. Interchain states, as we mentioned above, can be formed through exciton-exciton annihilation process if the exciton concentration is large enough, or via dissociation in the electric field. If the electric field is applied to the device, charge carriers are easily created at the expense of the interchain spaces.

In our theoretical model we first consider device without electric field. Under maximum illumination (the same as in Ref. [8]) from the monochromator we calculate the singlet exciton concentration profile in the active MEH-PPV film for the stationary case. We assume that every absorbed photon produces one singlet exciton (internal quantum efficiency of the exciton generation process is close to 1). By solving the continuity equation for singlet excitons we get the maximum value of the exciton concentration of $8.63 \times 10^{9} \mathrm{~cm}^{-3}$, which is much less than the approximate threshold $\left(10^{17} \mathrm{~cm}^{-3}\right)$ for exciton-exciton annihilation. Thus, we conclude that nonlinear secondary processes do not occur in our device. Since photocurrent increases rapidly with inverse bias voltage it is obvious that under the applied electric field the device operation is no longer based on exciton diffusion. Interchain species are generated by dissociation of singlet excitons and further, free carriers are produced. We assume that field-induced exciton dissociation is very fast, much faster than singlet exciton recombination $[5,7]$. The majority charge carriers emerged from the exciton dissociation are hole polarons because of the two orders of magnitude smaller electron polaron mobility $[8,9]$. Overall photocurrent of the biased photodiode is a sum of the excitonic and polaronic component. Polaronic photocurrent component is calculated in the same way as in Ref. [10] with linear generation and bimolecular recombination mechanism assumed. The fraction of excitons that undergo dissociation is field dependent [7]. To quantify the dependence of the polaron generation process on the applied electric field we compare simulated photocurrent spectra with experimental data. The coefficient that represents the fraction of excitons that have split to polarons (polaron generation coefficient) is tuned from 0 to 1 for every measured value of the bias voltage. The adequate value of the coefficient is determined by the best fit of the simulated curve to measured spectra. As the final result of our modeling we get the photocurrent density spectra that show excellent agreement with experiment for five different incident photon fluxes and bias voltages in the range from $0 \mathrm{~V}$ to $-10 \mathrm{~V}$. Also, the process of field-induced singlet exciton dissociation is quantified.

\section{Results and discussion}

Simulated and measured photocurrent density spectra are shown in Fig. 1 for three different bias voltages. The curves coincide very well in the whole wavelength range, accepting the slight disagreement for wavelengths between $400 \mathrm{~nm}$ and $500 \mathrm{~nm}$. All the data presented in Fig. 1 are derived with maximum photoexcitation intensity (the same as in Ref. [8]) from the monochromator. The similar agreement between the measurements and calculations is observed for the other four excitation intensities. Photocurrent density spectra are presented for maximum incident photon flux and high bias voltages because the experimental error is smaller for higher values of the measured current density.

The polaron generation coefficient presented in Fig. 2 is defined as the ratio of the number of secondary generated polarons to the number of the primary photogenerated singlet excitons. Figure 2 shows the polaron generation coefficient dependence on the electric field intensity. According to Fig. 2, for lower electric field intensities 


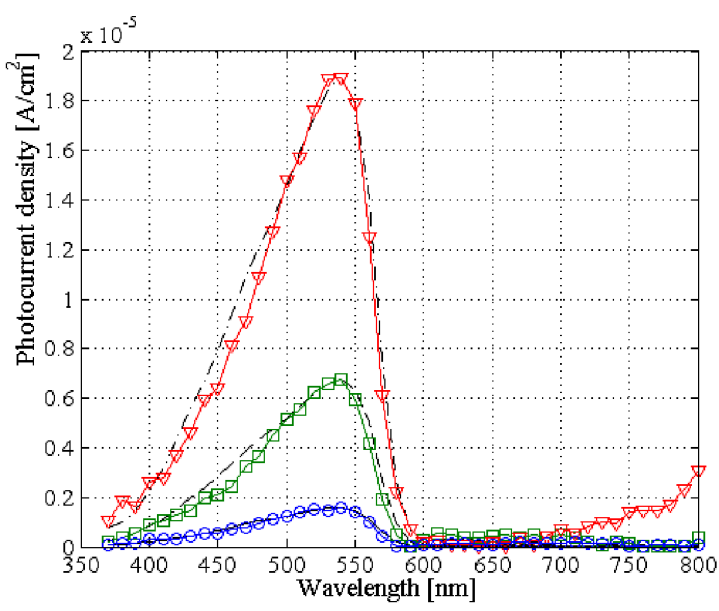

Fig. 1. Photocurrent density spectra of the ITO/PEDOT:PSS/MEH-PPV/Al photodetector. Curves with circles, squares and triangles represent measured data for bias voltages $-6 \mathrm{~V},-8 \mathrm{~V}$ and $-10 \mathrm{~V}$, respectively. Full black line, dashed line and dash-point-dash line represent simulation curves for $-6 \mathrm{~V},-8 \mathrm{~V}$ and $-10 \mathrm{~V}$, respectively.

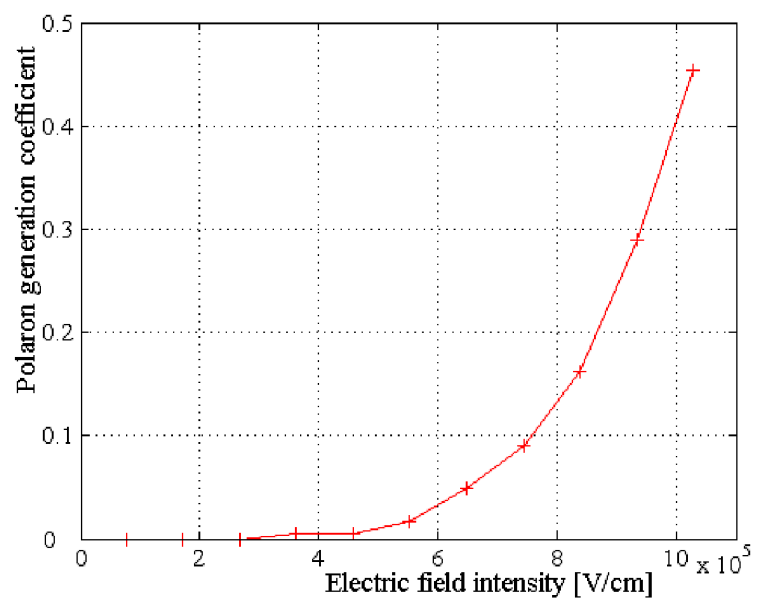

Fig. 2. Polaron generation coefficient versus electric field intensity.

$\left(<3 \times 10^{5} \mathrm{~V} / \mathrm{cm}\right)$ no polarons are generated in the bulk polymer.

With increasing electric field the number of polarons grows rapidly and reaches the value of 0.45 relative to the number of primary generated excitons, for the field intensity $\approx 10^{6} \mathrm{~V} / \mathrm{cm}$. The result is comparable to the work of Arkhipov et al. [7] in which they measure $30 \%$ of field-induced luminescence quenching for the electric field $\approx 2 \times 10^{6} \mathrm{~V} / \mathrm{cm}$. Luminescence quenching is explained in the light of the field induced exciton disso- ciation in the film of poly ( $p$-phenylphenylene vinylene) (PPPV) blended with polycarbonate (PC).

\section{Conclusion}

In this paper we have developed the ITO/PEDOT:PSS/MEH-PPV/Al photosensitive device model based on the singlet exciton diffusion and drift-diffusion of the hole polarons. Exciton-exciton annihilation process is not present in the device under illumination intensities used in our experiment. Hole polarons are generated through the field-induced exciton dissociation. Calculated photocurrent density spectra reproduce experimental data well for five different incident photon fluxes and in a wide range of reverse bias voltages. Field-induced exciton breaking is quantified through the polaron generation coefficient dependence on the electric field intensity. The results are in a good agreement with the literature.

\section{Acknowledgments}

This work was supported in part by the Serbian Ministry of Science under contract No. 16001 A, and in part by the Louisiana Experimental Program to Stimulate Competitive Research (EPSCoR), funded by the National Science Foundation and the Board of Regents Support Fund contract No. NSF (2008)-PFUND-111.

\section{References}

[1] G. Hadziioannou, P.F. van Huten, Semiconducting Polymers, Wiley-VCH, 1999.

[2] M.G. Harrison, J. Gruner, G.C.W. Spencer, Phys. Rev. B 55, 7831 (1997).

[3] B. Kraabel, V.I. Klimov, R. Kohlman, S. Xu, H.-L. Wang, D.W. McBranch, Phys. Rev. B 61, 8501 (2000).

[4] I.B. Martini, A.D. Smith, B.J. Schwartz, Phys. Rev. B 69, 035204 (2004).

[5] A.J. Lewis, A. Ruseckas, O.P.M. Gaudin, G.R. Webster, P.L. Burn, I.D.W. Samuel, Organic Electron. 7, 452 (2006).

[6] C.-X. Sheng, M. Tong, S. Singh, Z.V. Vardeny, Phys. Rev. B 75, 085206 (2007).

[7] V.I. Arkhipov, H. Bassler, M. Deussen, E.O. Gobel, R. Kersting, H. Kurz, U. Lemmer, R.F. Mahrt, Phys. Rev. B 52, 4932 (1995).

[8] J. Petrović, P. Matavulj, D. Qi, D.K. Chambers, S. Šelmić, IEEE Photon. Technol. Lett. 20, 348 (2008).

[9] J. Petrović, P. Matavulj, S. Šelmić, in: 51st ETRAN Conf. Proc., Herceg Novi-Igalo, 2007, Sect. MO.

[10] J. Petrović, P. Matavulj, L. Pinto, S. Šelmić, in: 53rd ETRAN Conf. Proc., Vrnjačka Banja, 2009, Sect. MO. 